\section{The chronic encephalopathy of Parry Romberg Syndrome and en coupe de sabre with a 31-year-history in a West Indian woman: clinical, immunologic and neuroimaging abnormalities}

\author{
Karan Seegobin, ${ }^{1}$ Kamille Abdool,,1,2 \\ Kanterpersad Ramcharan, 1,2 \\ Haramnauth Dyaanand,2,3 \\ Fidel Rampersad2,4
}

1Neurology Unit, San Fernando Teaching Hospital; 2University of the West Indies; ${ }^{3}$ Rheumatology Unit, San Fernando Teaching Hospital; 4Radiology Department, University of the West Indies, Trinidad and Tobago

\section{Abstract}

We describe a case of Parry Romberg syndrome/en coupe de sabre in a woman whose disease started as seizures at age 8 but was diagnosed at the age 39 . During these 31 years she got married, completed a first degree at university, had two successful pregnancies and has been gainfully employed. The features of generalized tonic-clonic seizures, autoimmune abnormalities, ocular abnormalities, morphea en coup de sabre and brain imaging abnormalities were present. Areas of parietal lobe cerebral calcification were encountered on the computed tomographic scan and bilateral periventricular white matter changes on the magnetic resonance imaging with frontal, temporal and parietal lobe brain atrophy ipsilateral to the facial hemiatrophy. Clinical, immunologic and neuroradiological abnormalities are discussed. In some cases, this illness can run a benign and stable course.

\section{Introduction}

Parry Romberg Syndrome (PRS) is an orphan or rare disease and is a poorly understood degenerative condition, seen most commonly as asymmetry of the face affecting dermatomes of one or multiple branches of the trigeminal nerve and rarely affects the limbs causing hemiatrophy. Rare cases of bilateral involvement have been described. Some authors refer to it as Parry Romberg Syndrome/en coupe de sabre (PRS/ECDS) since both conditions can occur separately or together. It most commonly presents in the first two decades of life and is commoner in females with an incidence of 0.3-2.5 cases per 100,000 population per year. ${ }^{1-4}$ PRS/ECDS can also be associated with neurologic, ophthalmologic, cardiac, rheumatologic, endocrinologic, infectious, orthodontic and maxillofacial manifestations and autoimmune findings. ${ }^{5}$ Whilst trophic malfunction of the sympathetic system has been proposed as the etiological basis, other reports suggest an autoimmune pathogenesis. ${ }^{2,6}$ Serological abnormalities in support of this putative mechanism have included the presence of antibodies to anticentromere, extractable nuclear antigen, rheumatoid factor, histone, Anticardiolipin, Anti Scl 70 and HLA B 27 antigens. 6,7 Recent evidence has suggested that there are diverse neurological presentations and late progression can occur with a fundamental immunologically mediated vascular pathogenesis with subcortical white matter changes on magnetic resonance imaging (MRI) suggestive of a chronic encephalopathic process. ${ }^{4-10}$ We report a case with a 3 decade history which highlights intracerebaral calcification and the bilateral white matter changes noted on T2-weighted and fluid -attenuated inversion recovery (FLAIR) MRI views and suggest this is typical of the periventricular hyperintensities seen in intracranial vascular disease of other etiologies inclusive of collagen vascular disease and referred to as leucoariaosis but not traditionally used in descriptions of this illness. ${ }^{8}$ The landmark study on the international epidemiology of PRS/EDCS by Stone reported no cases from the West Indies. ${ }^{11} \mathrm{We}$ document such a case.

\section{Case Report}

A 39-year-old woman of Indo-Trinidaian descent was referred for investigation of possible multiple sclerosis after periventricular white matter changes were noted on MRI of the brain following assessment for intermittent blurred vision of 8 weeks duration. At age 8 , she had noticed bald spots on her head, which were followed at age 11 by three dark lines that appeared on her forehead and right side of her face. At 18 years of age, she had her first partial seizure and experienced occasional headaches and intermittent visual loss, which lasted for a few seconds followed by generalized tonic-clonic seizures, which was managed with carbamazepine $400 \mathrm{mg}$ orally twice daily. She had approximately two to three seizures per year, which occurred only when she missed meals. At age 21, she noted upward deviation of her cheek-bones. After she had her first child at age 25, her chin deviated down and dark marks appeared at the middle of her chin and at the right side of her mouth.
Correspondence: Kanterpersad Ramcharan, Neurology Unit, San Fernando Teaching Hospital, San Fernando, Trinidad and Tobago.

Tel.: +1.868.342.6833 - Fax: +1.868.657.5951.

E-mail: kramcharan79@yahoo.com

Key words: Parry Romberg Syndrome; Rheumatology; Neuroradiology; Encephalopathy; West Indies.

Acknowledgements: we thank Miss Sharon Sealy for preparation of the images. We thank the patient for allowing us to report her clinical data.

Contributions: the idea for reporting this case was that of KA and KR. KS drafted the first draft of the manuscript. Further intellectual content and editing was done by KA, KR HD and FR. FR also provided advice on selection and interpretation of the radiological images. All authors saw, edited and approved the final contents. KR assumes responsibility for the integrity of the contents.

Conflict of interest: the authors declare no potential conflict of interest.

Received for publication: 25 June 2016.

Accepted for publication: 6 July 2016.

This work is licensed under a Creative Commons Attribution NonCommercial 4.0 License (CC BYNC 4.0).

(C)Copyright K. Seegobin et al., 2016 Licensee PAGEPress, Italy

Neurology International 2016; 8:6661

doi:10.4081/ni.2016.6661

She had no episodes of status epilepticus. Despite seeing many doctors of diverse specialties over the intervening years the PRS/ECDS was not recognized due to unfamiliarity with the entity. At age 39 , when she presented to us, she complained of blurred vision in the 2 preceding months, whenever she focused on objects. Her ophthalmologist assess ment reported anisocoria with the right pupil larger than the left (Figure 1A). Visual acuity was normal. In particular, there was no hypertension, diabetes, dyslipidemia, smoking, family history of intracranial, collagen vascular disease or stroke.

She went on to complete her secondary school education and then completed an Accounting degree. She had two children at the maternal age of 25 and 29 respectfully.

There was asymmetry of the right half of the face with atrophy of the muscles and a demarcation line between normal and abnormal skin. The right eye was mildly depressed in the socket, with asymmetry of the pupils and less prominent eyebrows. There was malar hypoplasia and atrophy of facial skin all confirmed by MRI. In addition, there was loss of 
subcutaneous fat with prominent bony ridges on the affected side and increased pigmentation on the ipsilateral upper forehead. There was atrophy of the right ala region of the nose (Figure 1A). A bald area can be seen on the scalp (Figure 1B). Other aspects of her clinical examination were normal. Based on this constellation of clinical features she was diagnosed with PRS/ECDS.

Investigations showed that hemogram, international normalized ratio, prothrombin time, partial thromboplastin time, renal, thyroid and liver function and urine analysis were normal. The antinuclear antibodies showed a fine speckled and homogenous pattern at 1/10 dilution: double stranded DNA antibody, rheumatoid factor, C3, C4, p and c antinuclear cytoplasmic antibodies, autoantibodies to extractable nuclear antigens, homocysteine and erythrocyte sedimentation rate were within normal limits. Cerebrospinal fluid analysis was normal with no oligoclonal bands. Abdominal ultrasound with doppler of renal arteries was normal. Echocardiogram was normal with an ejection fraction of $65 \%$. An interictal electroencephalogram was normal.

CT brain imaging showed areas of right parietal lobe hyperdensities consistent with calcification and mild atrophy of the right cerebral hemisphere as evidenced by prominence of the cerebral fissures and Sylvian fissure (Figure 2).

T1 FLAIR MRI axial views showed periventricular hyperintensities consistent with leukoariaosis more prominent in the right cerebral hemisphere with right frontal, temporal and parietal cortical atrophy (Figure 3A,B). Sagittal T2 FLAIR MRI view showed similar hyperintensities involving the corpus callosum (Figure 3C). Magnetic resonance angiogram of the brain and MRI spine were normal. Skin biopsy of an area of alopecia showed sclerosis

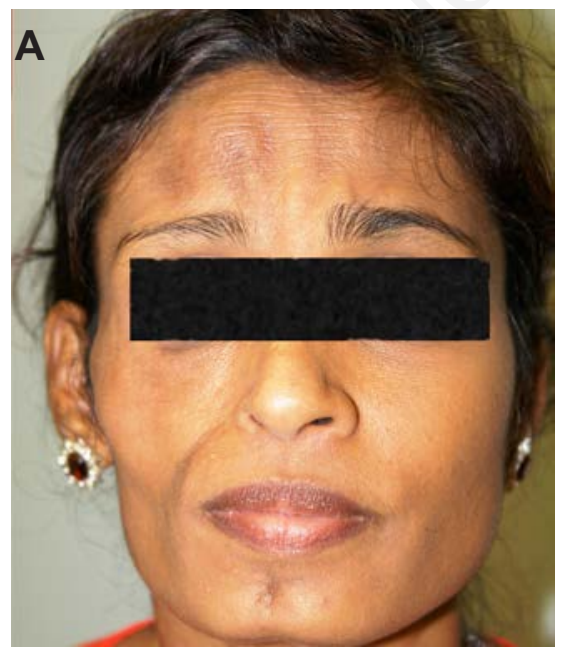

Figure 1. A) Showing right facial hemiatrophy and en coupe de sabre; B) showing bald patch on scalp.

of the dermis with normal adnexal structures and minimal inflammatory infiltrates of the mid-dermis.

\section{Discussion}

PRS has overlapping features of scleroderma en coup de sabre and the pathogenesis for both entities is unknown with no effective therapy available.12-14 While trophic malfunction of the sympathetic system has been proposed as a cause of PRS other reports suggest an autoimmune pathogenesis..$^{2,6}$

Whilst conflict exists between the differentiation of PRS from localized scleroderma, it is considered as a clinical subtype of linear scleroderma based on the recent literature and the classification of Mayo Clinic. 15 In en coup de sabre atrophy of underlying muscle or bone is not classically seen. Furthermore, there is prolonged nerve conduction in areas affected by scleroderma, which do not exist in PRS. Additionally, anti-nuclear anti-body titers are often raised with active linear scleroderma, but rarely so with PRS. ${ }^{16}$ While testing for immunological serologies has been done commonly in this condition, it is important to continue to do so as the presence of a positive rheumatoid factor or ANA is associated with increased risk of developing connective tissue

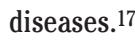

A positive ANA as in our case is the most common laboratory abnormality in PRS/ECDS, with approximately $25-52 \%$ of patients having an elevated titer. A theory supports that progressive hemi-facial atrophy (PHA) is an autoimmune condition. This is supported by the presence of other autoimmune conditions such as systemic lupus erythematosus (SLE), generalized myopathy, and rheumatoid arthri-

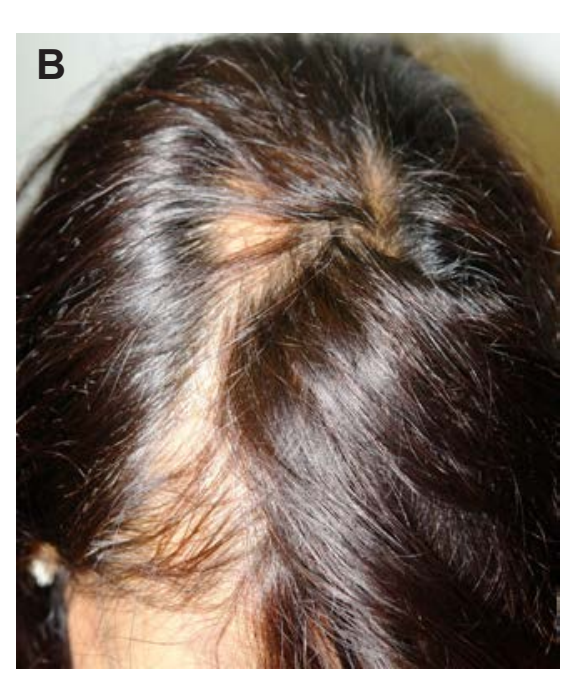

\section{be} the right cerebral hemisphere as evidenced etal lobe hyperdensities consistent with calcification and mild cerebral atrophy of by prominence of the cerebral and Sylvian fissures.

tis (RA) in some patients with PHA. In one study, 17 and $10 \%$ of surveyed respondents with PHA described a medical history of vitiligo and thyroid dysfunction, respectively. Other autoimmune conditions seen in these responders were inflammatory bowel disease (5\%), RA (4\%), ankylosing spondylitis (2\%), and SLE (2\%). ${ }^{7}$ On MRI, T2 hyperintensities is one of the most common finding in these patients suffering from PRS/ECDS, even with MRI evidence of microbleeds or microcalcifications. ${ }^{11,18,19}$ CT findings of calcification though rare have also been noted as in our case. ${ }^{20}$ In one report, it was noted that seizures were a common finding, however neurologic abnormalities were not correlated with the MRI imaging abnormalities. Furthermore the severity of superficial disease did not correlate with neurological symptoms or findings on neuroimaging. 18 Whether the MRI abnormalities have any correlation to serum immunological titers has not been previously documented. While the pathogenesis of neurological involvement is unclear, a vasculitic process has been suspected. In this report by Chiu et al., perivascular lymphocytic infiltrate with features of vasculitis was observed with ectatic blood vessels seen on both biopsies and angiothat vasculopathy or vasculitis are at the root

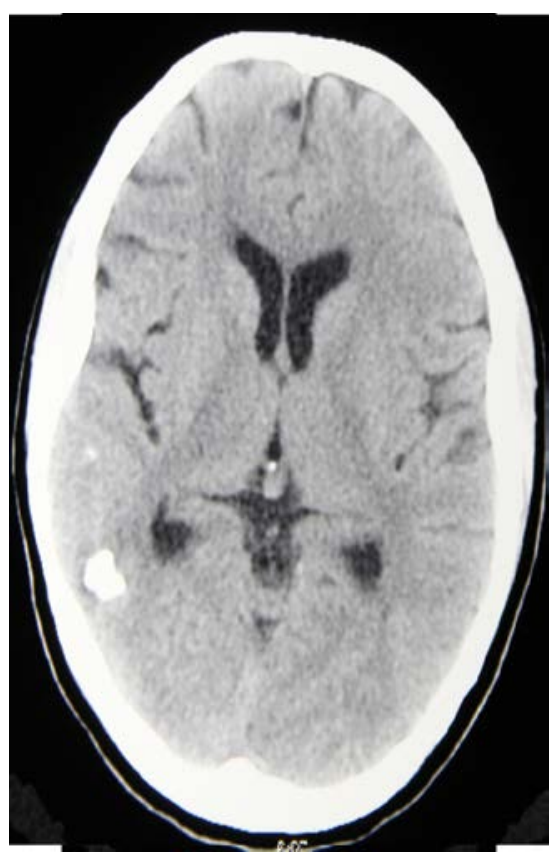
Figure 2. Axial computed tomography (n) graphic studies, which supported the theory 

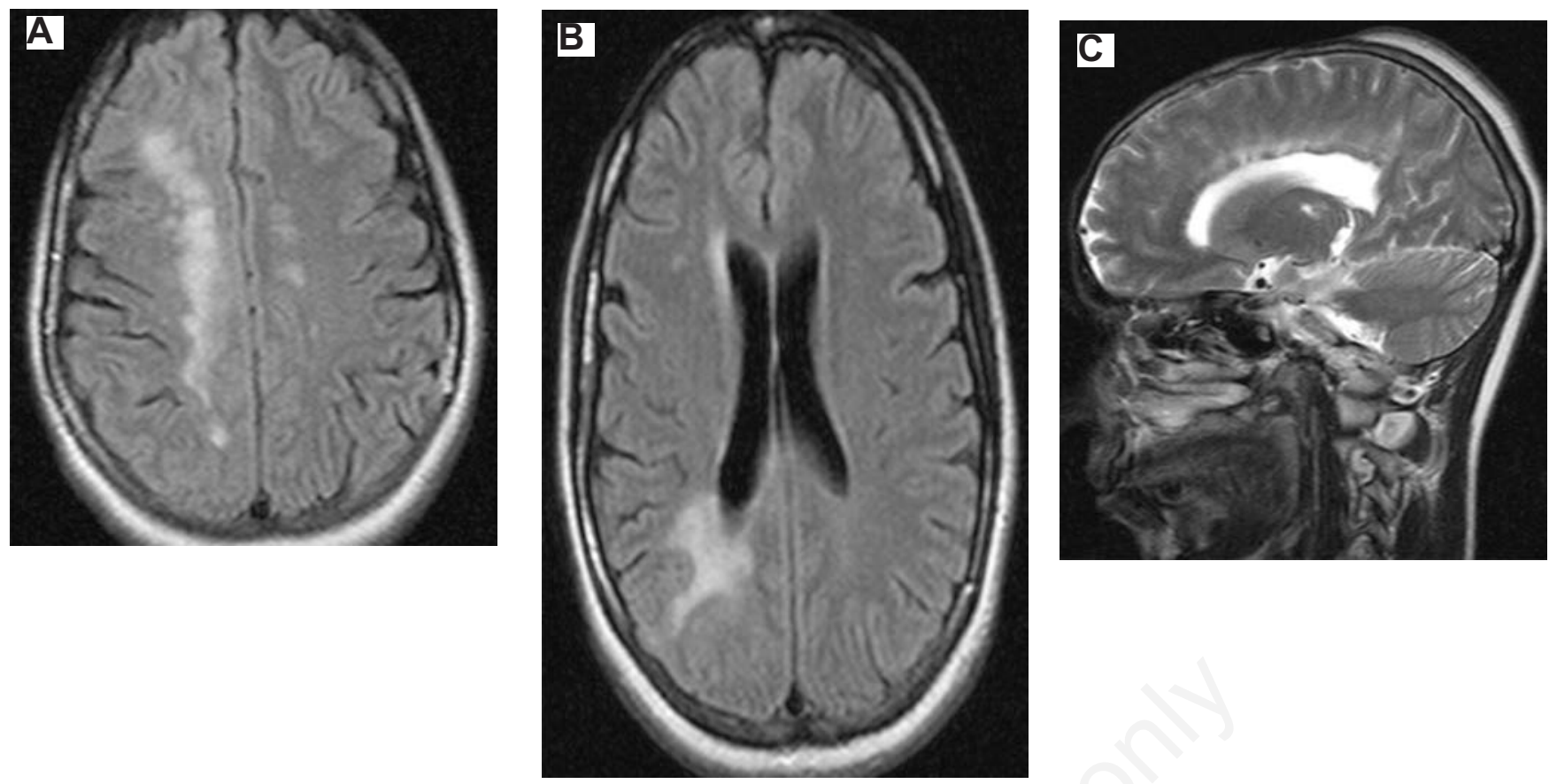

Figure 3. A) Axial T1 FLAIR magnetic resonance imaging (MRI) showing bilateral periventricular hyperintensities at supra ventricular level with temporal and parietal lobe cortical atrophy more prominent in the right cerebral hemisphere; B) axial T1 FLAIR MRI showing periventricular hyper intensities, consistent with a leukoariaosis type pattern and right frontal, temporal and parietal lobe cortical atrophy; C) sagittal T2 MRI showed involvement of the corpus callosum with T2 hyperintensities.

of the cerebral changes. 18 The presence of neurological involvement, hyperintensities on MRI and positive serologies of immunological parameters in our case, suggest that the underlying etiology for her condition is vasculitic. Other reports supporting this immunologic phenomenon have advocated research regarding the efficacy of immunosuppressants in these patients suffering from PRS. ${ }^{4}$ Brain histological studies in PRS/ECDS have been few and may be non-specific, consistent with changes of vasculitis. 4,10 Whilst the periventricular white matter changes in Figure $3 \mathrm{~B}$ in our case is radiologically similar to leucoariaosis described in other vascular disease, leucoariaosis is not a term used in descriptions of this illness and may be another observation for further examination. Definitive postmortem studies of the brain were not found and this is also an area of further research.

Despite the numerous neurological manifestations as summarized by Vix and colleagues in their recent review and late deterioration with a hemiparesis as noted by Dibaj and colleagues, in our patient apart from seizures, the illness has run a benign course. 4,10 Her care has been centered around seizure control and psychosocial support .

\section{Conclusions}

In conclusion, we report the first case of PRS/ECDS from the West Indies and highlight the typical clinical, serological and the rare neuroradiologic findings observed in larger case series. Stone recorded diagnostic delay up to 41 years in his global survey, 10 years longer than our patient. ${ }^{8}$ We document a stable natural history after 31 years of illness.

\section{References}

1. Rangare AL, Babu SG, Thomas PS, Shetty SR. Parry-Romberg syndrome: a rare case report. J Oral Maxillofac Res 2011;2:e5.

2. Madasamy R, Jayanandan M, Adhavan UR, et al. Parry Romberg syndrome: a case report and discussion. J Oral Maxillofac Pathol 2012;16:406-10.

3. Careta MF, Romiti R. Localized scleroderma: clinical spectrum and therapeutic update. An Bras Dermatol 2015;90:62-73.

4. Vix J, Mathis S, Lacoste $M$, et al. Neurological manifestations in ParryRomberg syndrome: 2 case reports. Medicine (Baltimore) 2015;94:e1147.

5. El-Kehdy J, Abbas 0, Rubeiz N. A review of Parry-Romberg syndrome. J Am Acad Dermatol 2012;67:769-84.
6. Garcia-de la Torre I, Castello-Sendra J, Esgleyes-Ribot T, et al. Autoantibodies in Parry-Romberg syndrome: a serologic study of 14 patients. J Rheumatol 1995;22:73-7.

7. Tolkachjov SN, Patel NG, Tollefson MM. Progressive hemifacial atrophy: a review. Orphanet J Rare Dis 2015;10:39.

8. Hâncu A, R anu I, Butoi G. White matter changes in cerebrovascular disease: leukoaraiosis. Available from: http://cdn.intechweb.org/pdfs/27263.pdf

9. Amaral TN, Marques Neto JF, Lapa AT, et al. Neurologic involvement in scleroderma en coup de sabre. Autoimmune Dis 2012;2012:719685.

10. Dibaj P, Herrendorf G, Bahn E, Obermann $M$. Late progression of neurological symptoms and MRI T2 hyperintensities in Parry-Romberg syndrome. J Neurol Neurosurg Psychiatry 2016. [Epub ahead of print]

11. Stone J. Parry-Romberg syndrome: a global survey of 205 patients using the Internet. Neurology 2003;61:674-6.

12. Jun JH, Kim HY, Jung HJ, et al. ParryRomberg syndrome with en coup de sabre. Ann Dermatol 2011;23:342-7.

13. Baskan EB, Kaçar SD, Turan A, et al. ParryRomberg syndrome associated with borreliosis: could photochemotherapy halt the progression of the disease? Photodermatol 
Photoimmunol Photomed 2006;22:259-61.

14. Brownell I, Soter NA, Franks Jr AG. Familial linear scleroderma (en coup de sabre) responsive to antimalarials and narrow band ultraviolet B therapy. Dermatol Online J 2007;13:11.

15. Khan M, Khan M, Negi R, Gupta N. Parry Romberg syndrome with localized scleroderma: a case report. J Clin Exp Dent 2014;6:e313-6.

16. Patel H, Thakkar C, Patel K. ParryRomberg syndrome: a rare entity. J
Maxillofac Oral Surg 2010;9:247-50.

17. Ouyang YH, Chuan MT, Yu HR, et al. Concurrent Parry-Romberg syndrome and systemic lupus erythematosus. Available from:

www.dermatolsinica.com/web/data/2008512023656.pdf

18. Chiu YE, Vora S, Kwon EK, Maheshwari M. A significant proportion of children with morphea en coup de sabre and ParryRomberg syndrome have neuroimaging findings. Pediatr Dermatol 2012;29:738-48.

19. Doolittle DA, Lehman VT, Schwartz KM, et al. CNS imaging findings associated with Parry-Romberg syndrome and en coup de sabre: correlation to dermatologic and neurologic abnormalities. Neuroradiology 2015;57:21-34.

20. Cory RC, Clayman DA, Faillace WJ, et al. Clinical and radiologic findings in progressive facial hemiatrophy (Parry-Romberg syndrome). AJNR Am J Neuroradiol 1997;18:751-7. 